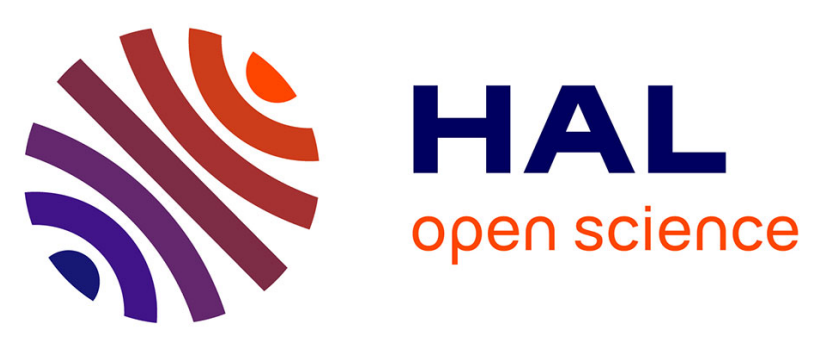

\title{
Arbres fruitiers et cultures jardinées gallo-romains à Longueil-Sainte-Marie (Oise)
}

Philippe Marinval, Denis Maréchal, David Labadie

\section{To cite this version:}

Philippe Marinval, Denis Maréchal, David Labadie. Arbres fruitiers et cultures jardinées gallo-romains à Longueil-Sainte-Marie (Oise). Gallia - Archéologie de la France antique, 2002, 59, pp.253-271. 10.3406/galia.2002.3052 . hal-01909332

\section{HAL Id: hal-01909332 \\ https://hal.science/hal-01909332}

Submitted on 20 Jan 2020

HAL is a multi-disciplinary open access archive for the deposit and dissemination of scientific research documents, whether they are published or not. The documents may come from teaching and research institutions in France or abroad, or from public or private research centers.
L'archive ouverte pluridisciplinaire HAL, est destinée au dépôt et à la diffusion de documents scientifiques de niveau recherche, publiés ou non, émanant des établissements d'enseignement et de recherche français ou étrangers, des laboratoires publics ou privés.

\section{(ㅇ)(1) $\$$}

Distributed under a Creative Commons Attribution - NonCommercial - NoDerivatives| 4.0 


\title{
ARBRES FRUITIERS ET CULTURES
} JARDINÉES GALLO-ROMAINS À LONGUEIL-SAINTE-MARIE (OISE)

\author{
Philippe MARINVAL , Denis MARÉCHaL ${ }^{* *}$ et David LABADIE*
}

\begin{abstract}
Mots-clés. Picardie, Gaule, Gallo-Romain, arboriculture, horticulture, carpologie.
Key-words. Picardie, Gaul, Gallo-Roman, arboriculture, horticulture, carpology.

Résumé. Cét article présente plusieurs remarquables déconvertes de vestiges archéobotaniques prozlenant d'un site gallo-romain de la moyenne vallée de l'(Oise : Le Bois Harlé-La Queue de Rivecourt à Longueil-Sainte-Marie (Oise). Il s'agit notamment de restes de fruits de Gin pignon (Pinus pinea) et de gourde calebasse (Lagenaria siceraria), ainsi que de graines de bette/betterave (Beta vulgaris) et de buis Buxus sempervirens). Ces vestiges permettent de sinterroger sur les lieux de production el témoignent du dynamisme de l'arboriculture. ot de l'horticulture gallo-romaines.

11)

bstract. In this article some plants material which came out of a Gallo-Roman site of the middle valley of the River Oise Le Bois Harle1. Queue de Rivecourt al Longueil-Sainte-Marie are being examined. It consists particularly of fruit remains from the stome pine (Pinus Dinea) and the gourd (Lagenaria siceraria), and of beet (Beta vulgaris) and box (Buxus sempervirens) seeds. It is now possible to analyse the areas of cultivation from these remains which provide a good evidence of the dynamism of arboriculture and horticulture in Roman Gaul.
\end{abstract}

\section{LE SITE}

L'équipe de recherche archéologique du CRAVO effectue depuis des années des sauvetages sur les sites archéologiques menacés par les sablières et gravières de la moyenne vallée de l'Oise. À cette occasion, elle a mené des travaux d'urgence sur des gisements de la commune de Longueil-Sainte-Marie (Oise), notamment sur un ensemble gallo-romain situé à cheval sur deux lieux-dits : Le Bois Harlé et La Queue de Rivecourt.

Le site est localisé au nord-est du Bassin parisien (fig. 1), à $12 \mathrm{~km}$ au sud-ouest de Compiègne, dans la moyenne vallée de l'Oise (département de l'Oise). Implanté sur la rive droite de la rivière, à $1300 \mathrm{~m}$ de son cours actuel, il occupe la première terrasse de

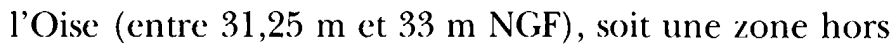
d'atteinte des crues annuelles actuelles.

À moins de $600 \mathrm{~m}$ au sud-est, s'écoule un ruisseau qui occupe le centre d'une large dépression correspondant à un ancien chenal lateral de l'Oise (probablement tardiglaciaire). Un second cours d'eau délimite cette terrasse au nord-est. Il prend naissance au pied de la buttetémoin éocène : la Montagne. Il a déposé un cône de sédimentation qui a engendré le colmatage d'une partie

* Centre d'Anthropologie, UMR 855\% du CNRS, EHFSS, Lniversité Paul-Sabatier, Lniversité de Toulouse-Le Mirail, 39 allées Jules-(suesde, F-31000) Toulouse.

** CRAVO/INRAP, Base archéologique, 526 rue des Lombards, F-60680 I.e Fayel. 




Fig. 1 - Localisation et plan de la fouille du site gallo-romain du Bois Harlé/La Queue de Rivecourt à Longueil-Sainte-Marie, Oise (I)AO D. Maréchal, INRAP).

de la frange septentrionale de la plaine alluviale. Cette dynamique a également contribué à colmater les dépressions correspondant aux anciens chenaux latéraux qui limitent la terrasse au sud-ouest.

La surface décapée de 1988 à 1993 couvre 47 ha répartis entre les parcelles du Bois Harlé ( 13 ha), de La Queuc de Rivecourt (1 ha), qui correspondent au centre du village, et celles du Bois à Bouleau (16 ha) et du Petit Muid (1,2 ha) qui constituent sa périphérie (fig. 2).

La fouille a mis au jour 3800 structures archéologiques, dont 400 fossés ou tronçons particls. La stratégie de fouille, tributaire des délais imposés par l'exploitation des carriers, a dû adapter les moyens disponibles aux objectifs. La plupart des fosses (75\%) ont été fouillées sur une moitié, les fossés étant, eux, sondés régulièrement (sauf certains tronçons fouillés intégralement sur Le Bois Harlé). La quantité de mobilier mis au jour, qui avoisine les $2 \mathrm{t}$, ne représente donc, au minimum, qu'un cinquième de la masse totale.

Cette opération d'assez grande envergure a permis d'appréhender le noyau villageois et ses parcelles périphériques. Fondée vers 70 après J.-C., l'agglomération est implantée sur la terrasse dominant la plaine inondable de l'Oise (fig. 1 et 2). Le nombre élevé de fossés mis au jour accrédite l'importance du facteur hydrique dont la pérennité a permis une bonne conservation des sédiments dans les creusements profonds (Bernard, Dietrich, 1990). L'arrêt de l'entretien de ces fossés au début du III's. précède de peu l'abandon définitif du site.
L'étude de la céramique montre la présence de formes sigillées de la Gaule du Sud, du Centre et de l'Est, selon le schéma le plus classique (fig. 3 et 4 ). Les différents ateliers du sud ( $\mathrm{I}^{\mathrm{er}} \mathrm{s}$.) mais surtout de l'est et du centre de la France (II's.) se concurrencent. À la fin du II" s., ce sont finalement les produits orientaux qui prédominent. On trouve aussi les autres types de céramique fine, mais toujours en faible proportion. Ainsi la terra nigra qui occupe une place privilégiée à la fin du $\mathrm{I}^{\mathrm{er}}$ et au début du II ${ }^{*}$ s. disparaît progressivement. Le même phénomène s'observe pour les céramiques dorées au mica qui disparaissent au profit de celles à enduit rouge à partir du milieu du II" s. Les vases décorés sont peu nombreux, excepté durant le $\mathrm{I}^{\mathrm{er}} \mathrm{s}$.

Cependant, l'essentiel du mobilier est d'origine locale. Progressivement les vases à pâte blanche sableuse vont s'imposer pour accéder à un quasi-monopole au III" $s$.

Globalement, il ressort une impression de vie en autarcic de cette communauté, bien que des échanges, surtout avec les régions avoisinantes dans un rayon de $100 \mathrm{~km}$, soient attestés (Pissot et al., 1994).

Les études entreprises sur le mobilier comme sur l'architecture indiquent un statut social modeste des habitants, dont les activités sont surtout agropastorales (Lepetz, Maréchal, 1996 ; Pinard et al., 1999). Ces caractéristiques associées à l'ordonnancement et l'organisation spatiale du site définissent une occupation que l'on peut qualifier de villageoise. 

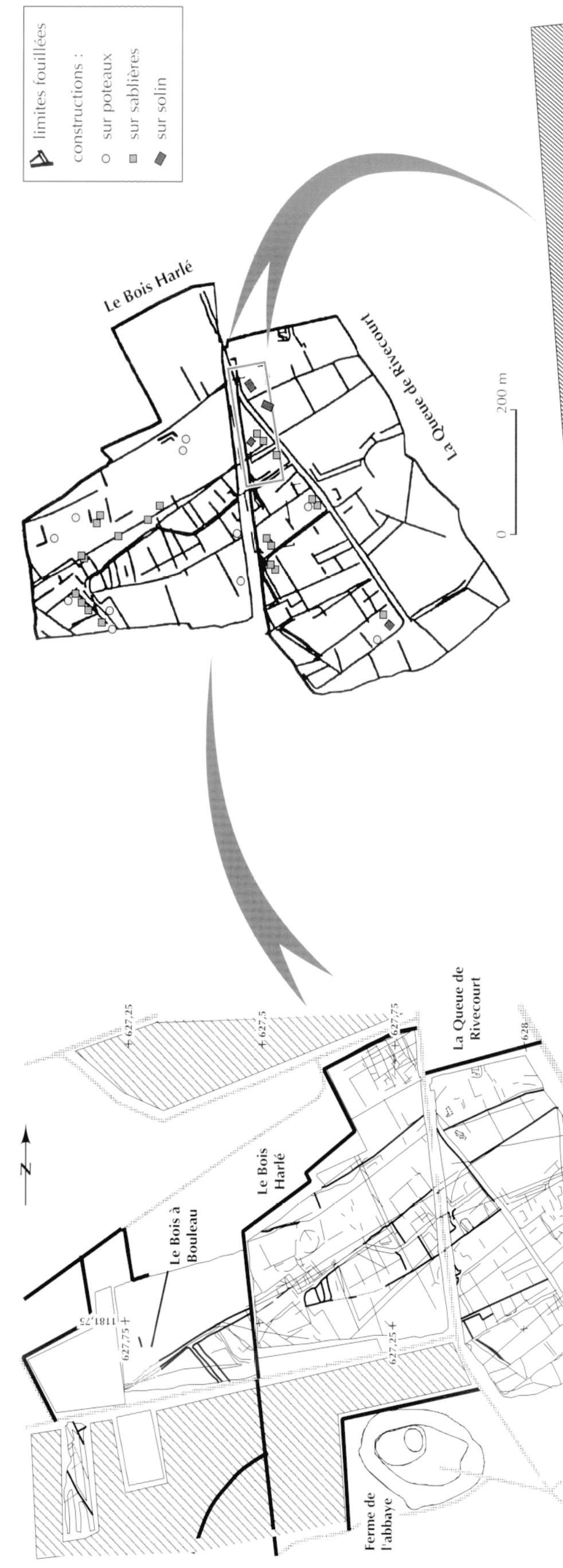

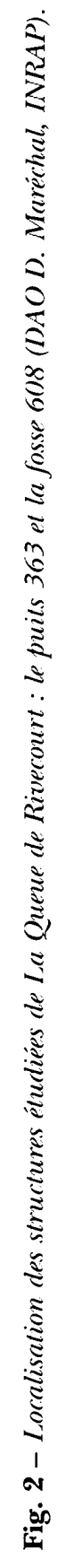



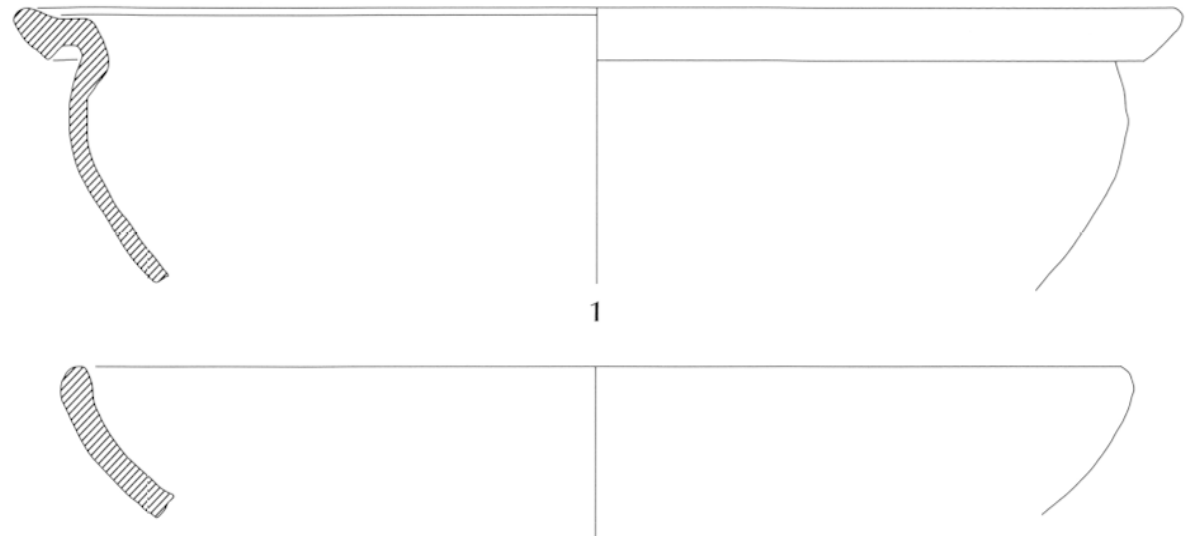

2
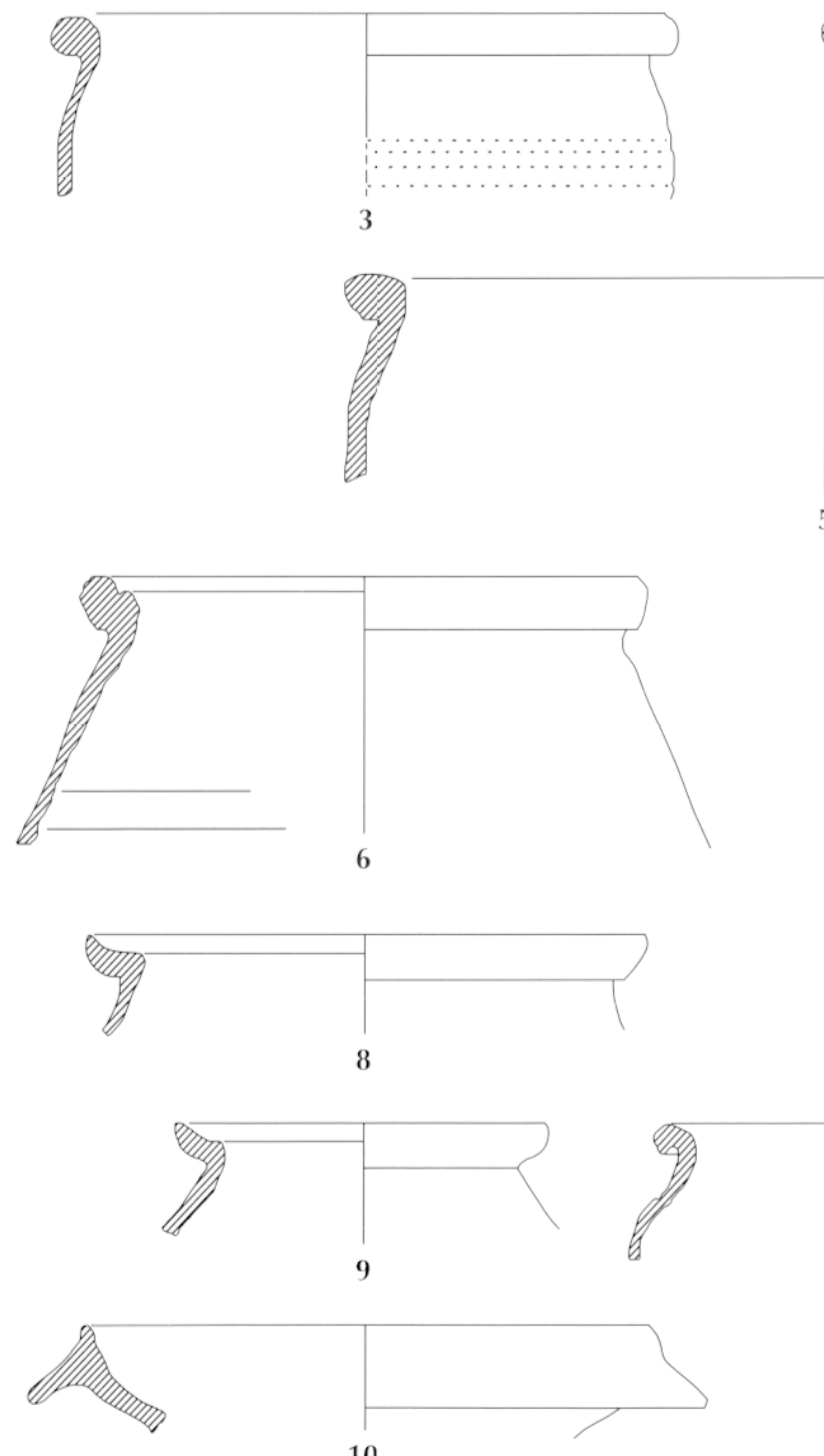

10 $5 \mathrm{~cm}$

0
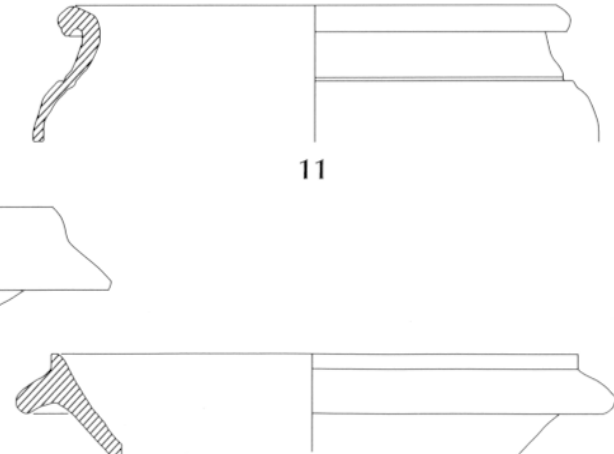

11

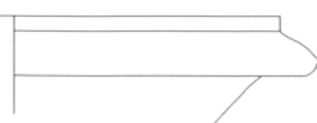

12
5

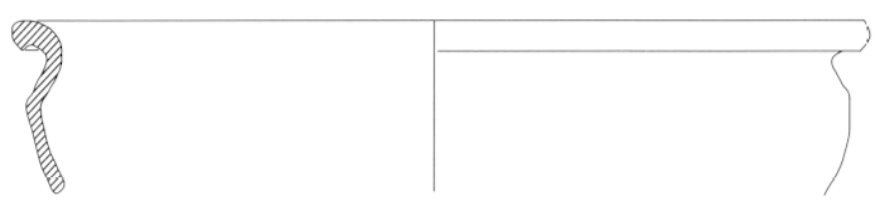

4
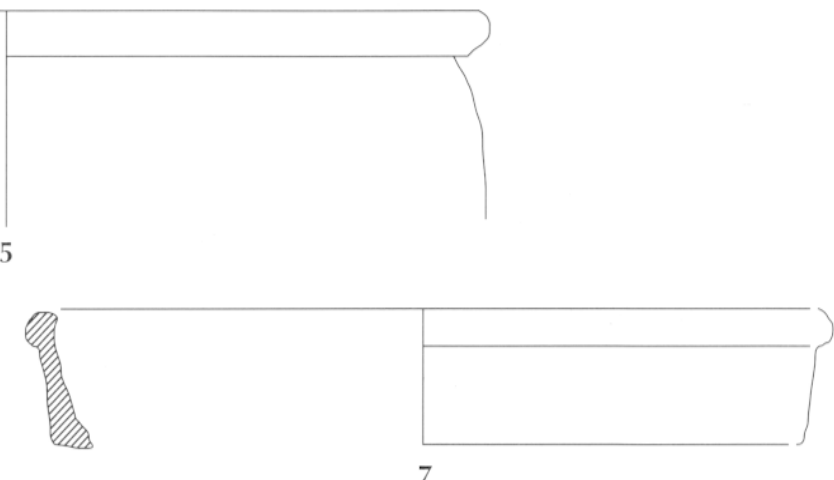

7

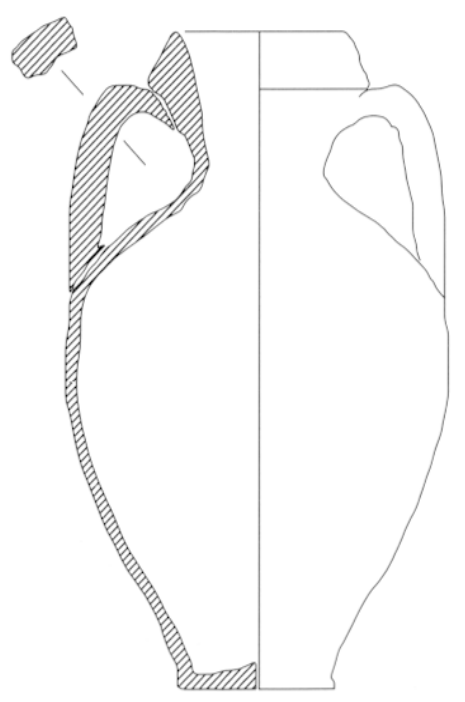

13

Fig. 3 - Córamique commune recueillie au Bois Harlé/I.a Queue de Riverourt (dessin I). Maréchal, INRAP). 

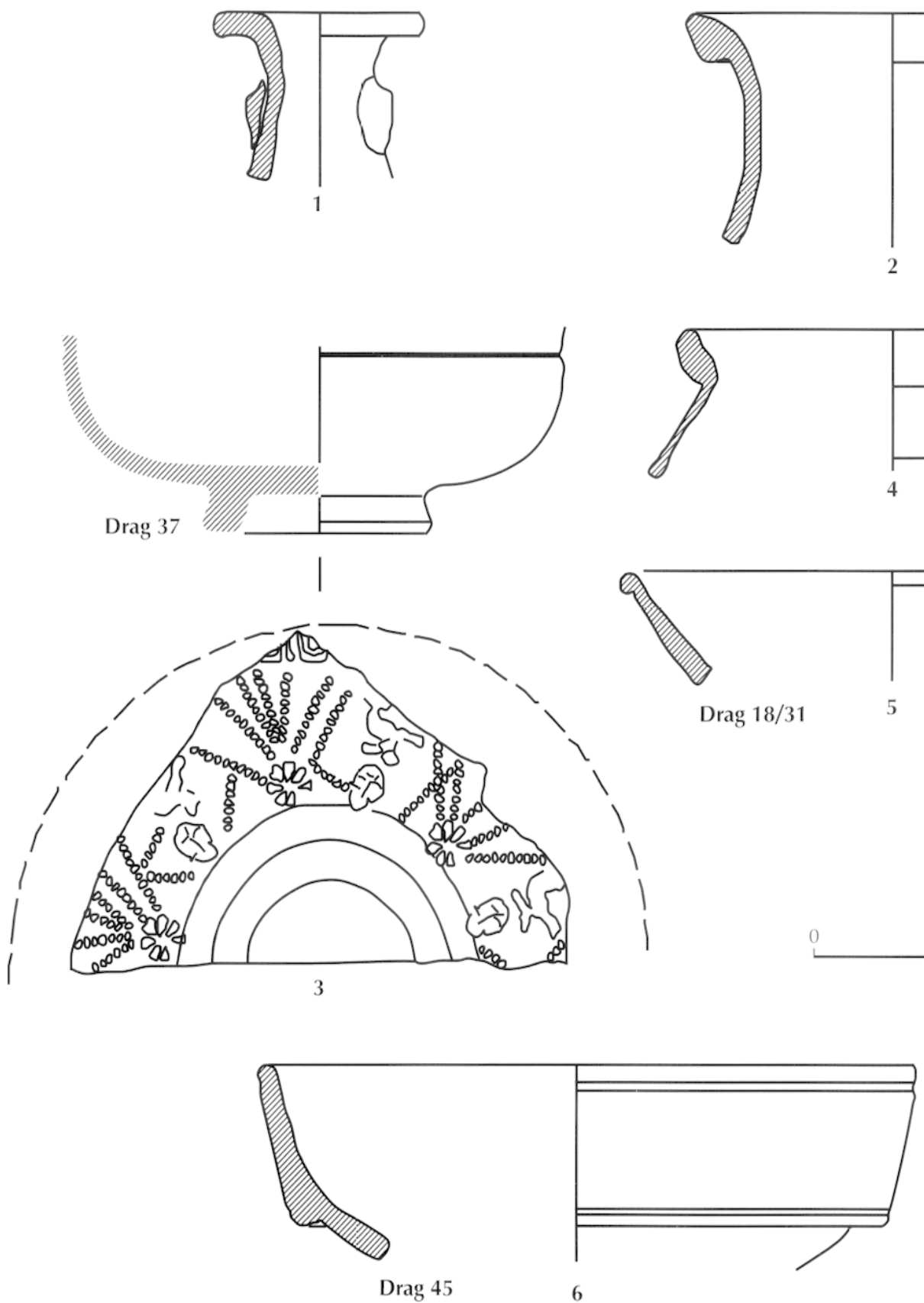

Fig. 4 - Céramique sigillée recueillie au Bois Harlé/La Queue de Rizerourt (dessin D. Maréchal, INRAP).

\section{LES RECHERCHES CARPOLOGIQUES}

Au cours de ces investigations, lors de décapages à la truelle, des paléo-semences ont été mises au jour. Un grand nombre de prélèvements de sédiment, en vue d'analyses carpologiques, a également été réalisé. Ceuxci ont livré de nombreux carpo-restes. Quelques-uns sont carbonisés, mais, pour l'essentiel, ils se sont conservés
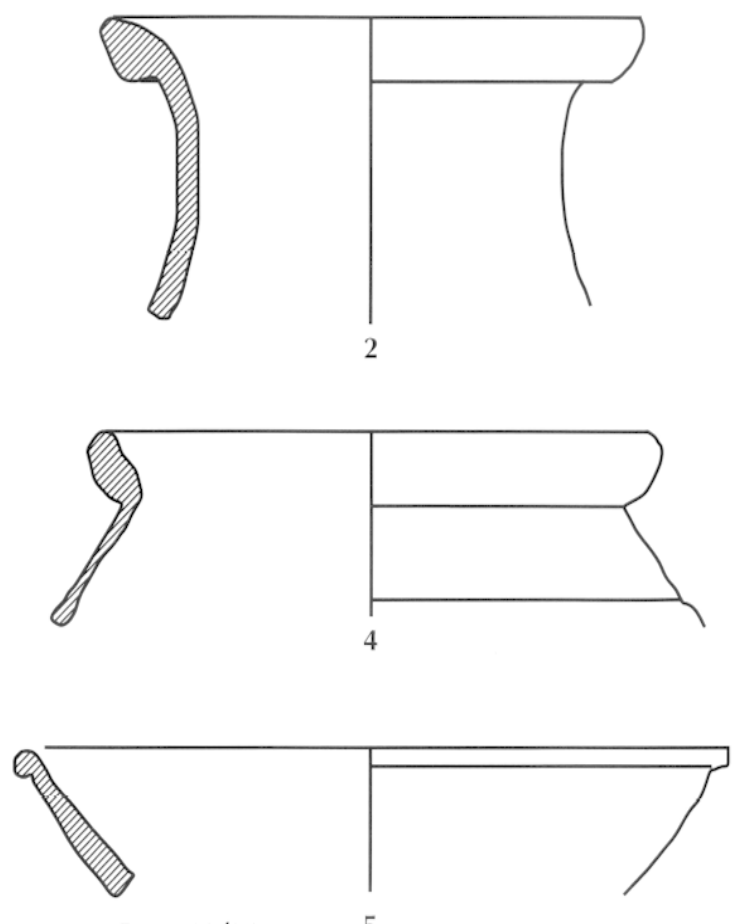

Drag 18/31

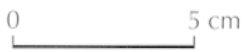




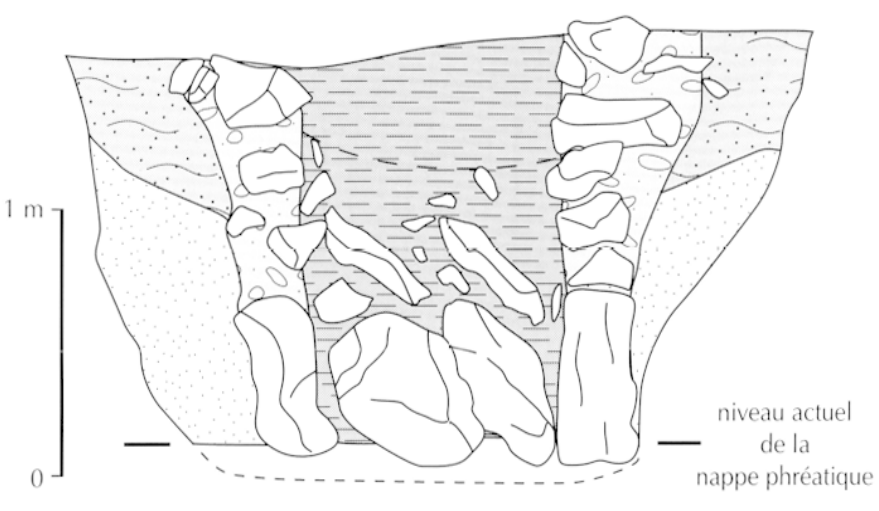

sédiment argileux, gris foncé + oxydation

sédiment sableux légèrement argileux, gris foncé

sédiment très sableux

sédiment sableux

Fig. 5 - Coupe du puits 363 de La Queue de Riveconrt (I)AO D). Maréchal, INRAP).

des plantes cultivées attestées sur le site (De Hingh, 1993). P. Marinval a publié les restes de pigne de pin pignon et du fruit de cucurbitacées dans le cadre d'un article grand public consacré à l'alimentation végétale gallo-romaine (Marinval, 1992).

Cet article présente deux découvertes remarquables effectuées sur la parcelle voisine: La Queue de Rivecourt, ainsi que les résultats carpologiques provenant de deux structures, le puits 363 et la fosse 608 (fig. 2).

\section{LE PUITS 363}

Ce puits possède une profondeur d'un peu plus de $1,50 \mathrm{~m}$. Son assise est constituée de gros blocs de grès sur lesquels reposent des pierres et des dalles de gabarit plus petit (fig. 5). Le comblement ultime, argileux, avec des traces d'oxydation, est daté par le mobilier de la seconde moitié du II"s. après notre ère. Cette structure se localise au sein d'un ensemble dense de fosses, associé à des constructions sur sablières constituant de probables habitats.

Dans le comblement du puits a été recueilli un cône de pin pignon (Pinus pinea l..) qui est bien conservé. Il présente une longueur de $88 \mathrm{~mm}$ et une largeur maximale de $61 \mathrm{~mm}$. Il est à moitié écalé. Les bractées ou écailles ne subsistent que dans la partie supérieure du cône (partie la plus éloignée du pédoncule) (fig. 6).

\section{ARCHÉOLOGIE DU PIN PIGNON (PINUS PINEA)}

Le pin pignon, pin pinier ou bien encore pin parasol est un arbre de la zone méditerranéenne (il s'étend naturellement du Portugal à la Syrie). Sa silhouctte est si caractéristique qu'elle constitue pratiquement un emblème de cette région (Rol et al., 1981). Les cônes qui contiennent chacun une centaine de graines mûrissent en automne-hiver de la troisième année.

L'arbre est largement entretenu ou planté, depuis au moins l'Antiquité, notamment en Italie à des fins culinaires, décoratives, voire rituelles (André, 1981). Les cônes furent fréquemment représentés dans l'iconographie (peinture murale, mosaique...) et la sculpture romaines.

Comme l'attestent les données textuelles (Pline, Columelle, Apicius, Celse, Palladius... cités par J. André, 1981), les pignons ou graines, oléagineux au goût agréable, furent abondamment consommés sous différentes formes (fruits, condiments...). Ils entraient dans la composition de nombreuses recettes de cuisine comme en témoignent les fréquentes mentions dans les écrits d'Apicius (L'arl culinaire). Les pignons étaient, semble-t-il, récoltés avant leur complète maturité afin que la dispersion naturelle des graines (ouverture par déhiscence des écailles) ne s'opère pas. Ils pouvaient ainsi être stockés jusqu'à l'été.

Les découvertes en contexte archéologique de cônes ou de pignons carbonisés ou imbibés sont assez fréquentes. Elles s'échelonnent des régions méditerranéennes à l'Angleterre et aux Pays-Bas (voir notamment : Willcox, 1977 ; Meyer, 1980 ; Green, 1981 ; Coulon et al., 1985 ; Kislev, 1988 ; Ruas, 1990 ; Marinval, 1993 ; De Hingh, Kooistra, 1995; Matterne, 2001). En Europe occidentale, elles datent presque toutes de l'époque romaine, si bien que la mention d'un vestige de l'espèce constitue pratiquement un fossile directeur pour la période.

Le pin pignon pousse actuellement et fructifie même en région parisienne. Depuis des années, de beaux individus fournissent ainsi des cônes fertiles dans l'arboretum de Chèvreloup, Yvelines (renseignements Service des cultures du Muséum national d'histoire naturelle). On peut donc fort bien estimer que cet arbre a pu être 


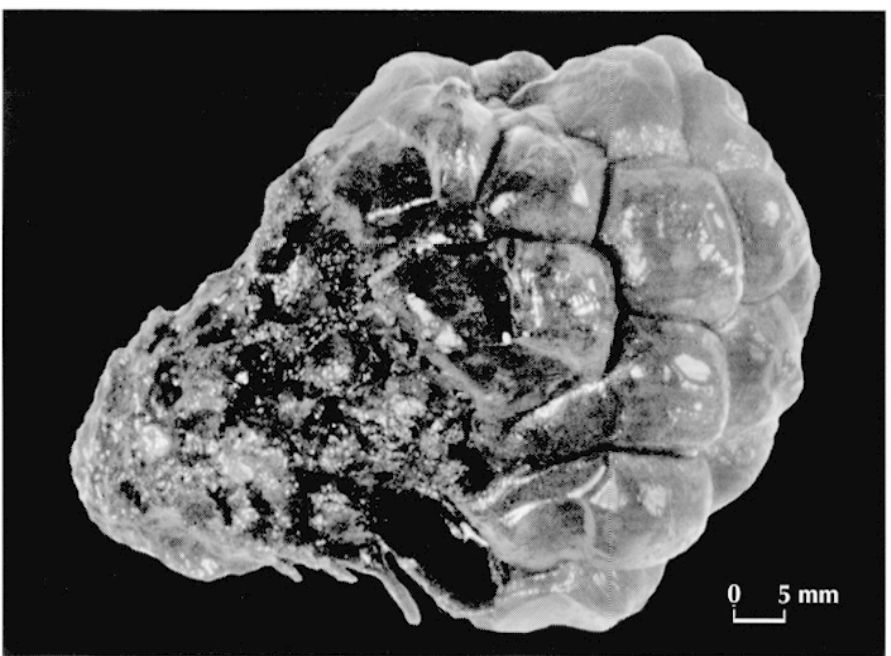

Fig. 6 - Le cône de pin pignon (Pinus pinea) du puits 363 de La Queue de Rizecourt. On remarque qu'il est à moitié écalé (photo A. (jue), (NRS).

acclimaté et croître à l'époque romaine dans l'Oise. Des collègues britanniques pensent qu'il en était de même en Angleterre (Green, 1981). Toutefois, l'importation de produits en provenance de la zone méditerranéenne n'est évidemment pas à exclure. Au reste, le transport de cônes par voie maritime est attesté. Les épaves d'au moins deux navires romains en recelaient. Des exemplaires figuraient en fond de cale dans le bateau de la madrague de (iens, Var (Girard, Tchernia, 1978), d'autres étaient coincés dans le col d'amphores vinaires dans le navire qui a sombré au large d'Albenga, Ligurie (Lamboglia, 1952). L'exportation d'autres produits exotiques, olives, figues, dattes en Europe du Nord durant la période romaine est d'ailleurs bien documentée (Willcox, 1977 ; Green, 1981 ; Greig, 1991 ; Knörzer, 1991, Bakels, 1991 ; Marinval, 1993).

Les contextes de découvertes des vestiges archéologiques du pin pignon indiquent au moins trois types d'utilisation des productions de l'arbre.

- Les trouvailles dans les unités domestiques, comme certaines habitations de Pompéi ou d'Herculanum (Meyer, 1980) ou de Gaule, par exemple dans la cave de l'habitat rural du Tremblay (Val-de-Marne) où l'on a retrouvé des écailles et des valves carbonisées (Matterne, 2001), attestent la consommation courante des pignons. - La présence de cônes dans les foyers-autels de temples dédiés à Mithra à Londres (Willcox, 1977), à Cybèle à Alba-la-Romaine en Ardèche (Marinval, inédit), à Isis à Belo en Andalousie (Lignereux et al., 1997) témoigne de leur utilisation comme offrandes votives. Les cônes étaient brûlés dans les foyers lors du rituel de célébration des divinités.

- Les nombreuses découvertes de pignons ou de fragments de cônes carbonisés dans les sépultures à incinération de Gaule romaine démontrent l'importance de ce fruit dans le domaine funéraire (Marinval, 1993; Pradat, 1994). Il devait être placé en compagnie d'autres offrandes sur le bûcher au moment de la crémation du défunt.

Pour les anciens, l'arbre était considéré comme un symbole de fertilité et de longévité exemplaire (Brosse, 1989, 1990). Aussi, n'est-il pas surprenant que ses fruits aient été associés à la pratique de cultes où la sexualité occupait une large place ainsi qu'au monde des morts. D'autres cônes de pin pignon sont signalés en Gaule. Dans les sédiments grecs du port de Marseille (Bouby, Marinval, 2000) ainsi que dans des puits romains du Haut-Empire à Saintes (Charente-Maritime) (Collectif, 1980), à Jouars-Pontchartrain (Yvelines) (Matterne, 2001), à Chartres (Eure-ct-Loir) et à Rodez. (Aveyron) (les deux dernières Marinval, inédit).

L'exemplaire de Chartres est tout à fait similairc à celui de Longueil-Sainte-Marie. Il se présente également à demi-décortiqué (Marinval, inédit). La position des cônes de pin pignon au sein du remplissage des puits et l'absence d'une partie de leurs écailles laissent à penser que, dans les deux cas, il s'agit de déchets culinaires. Après consommation d'une partie des pignons, les cônes furent rejetés comme d'autres déchets dans le puits. Toutefois, la raison de l'arrêt de leur consommation et de leur élimination nous échappe ; d'autant plus que des pignons, apparemment bien formés, sont observables lorsqu'on écarte légèrement les écailles qui subsistent sur les deux cônes.

\section{LA FOSSE 608}

Elle est placée $165 \mathrm{~m}$ plus au nord que le puits 363 et se situe à proximité de petits thermes privés. Flle a empiété sur des fossés plus anciens. Longue de 7,20 m, elle est constituée de deux parties distinctes. Au sud, un creusement quadrilatère de $3,20 \mathrm{~m}$ sur $2,80 \mathrm{~m}$ et profond de $1,48 \mathrm{~m}$ a conservé une ossature de piquets entre lesquels ont été disposés, sans grand soin, des fagots de brindilles. Le trapèze ainsi dessiné ressemble, de fait, à un puits. Au nord-ouest, le second creusement, moins 

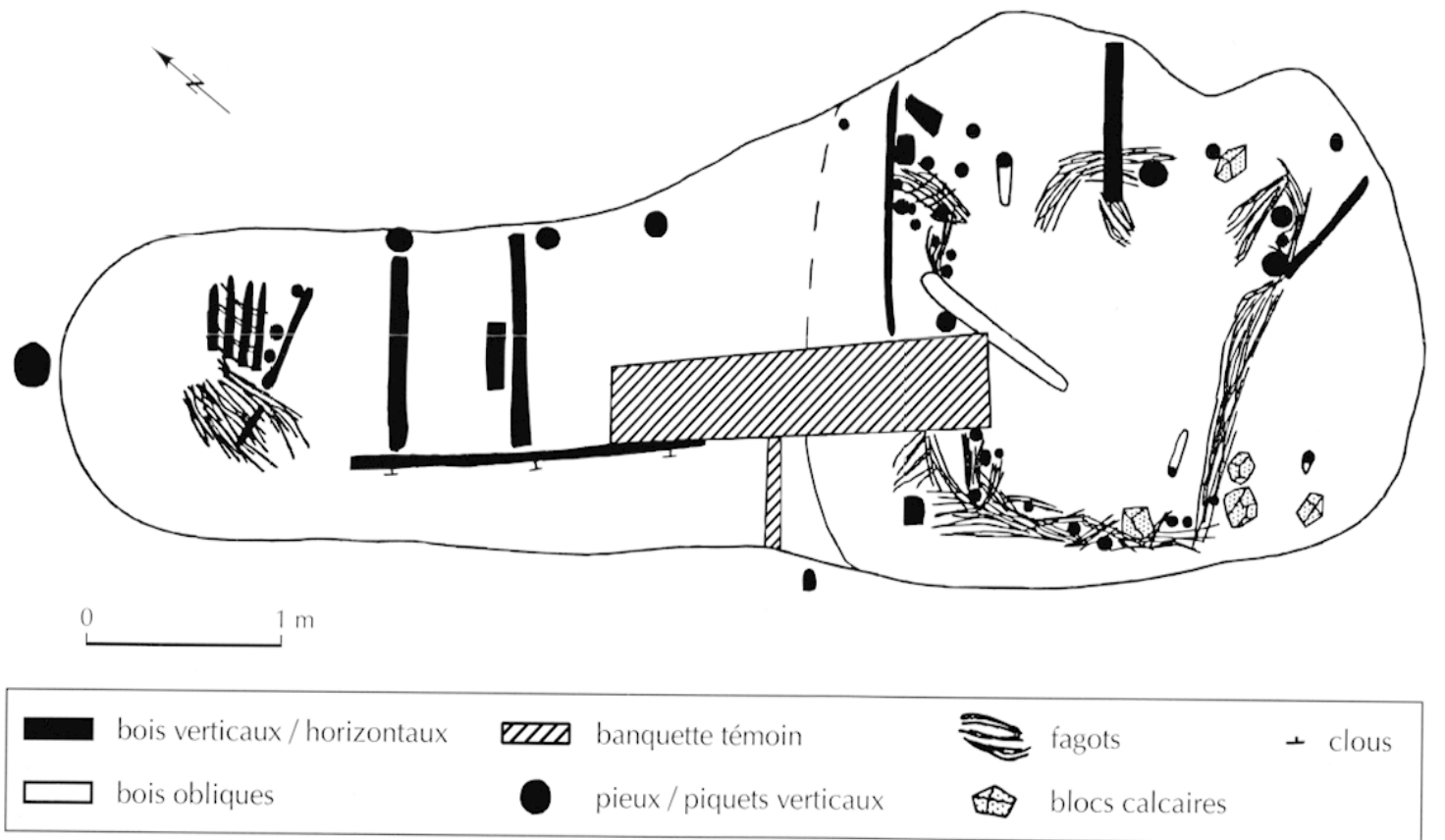

Fig. 7 - Plan de la fosse 608 de La Queue de Rivecourt (relevé A. (iuey, CNRS).

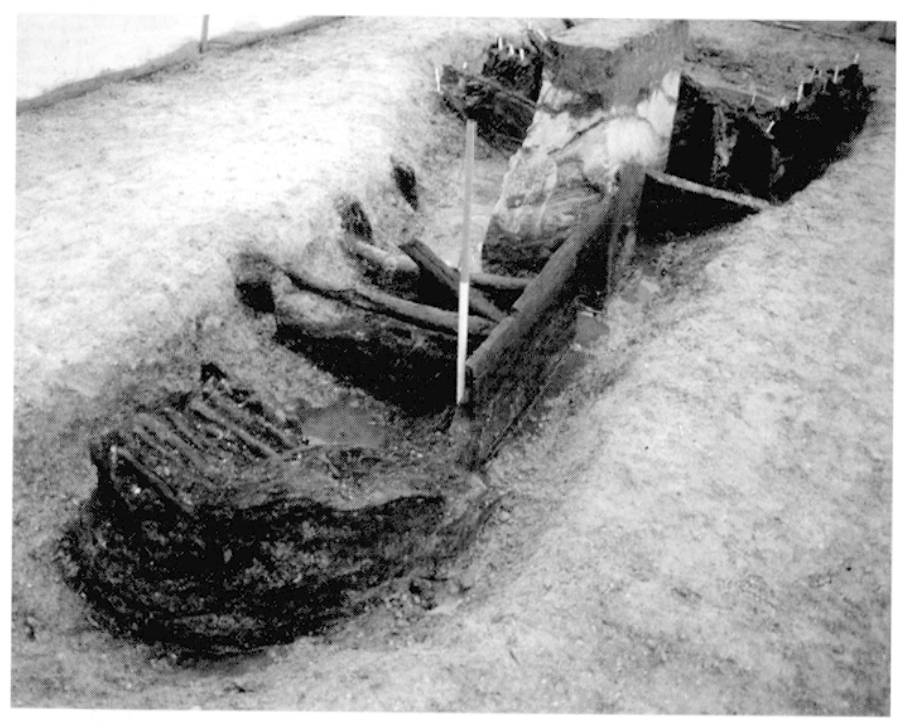

Fig. 8 - Vue de la fosse 608 de La Queue de Rivecourt (photo A. (sury, CNRS).

profond $(1,10 \mathrm{~m}$ max.) et plus étroit $(1,50 \mathrm{~m})$, recèle un aménagement particulier fait d'une longue planche en chêne verticale et de deux traverses au minimum (une troisième a dû disparaître) perpendiculaires, associées à des poteaux (fig. 7 et 8). À l'origine, elles devaient être clouées à la planche à des niveaux différents. Le remplissage de cette seconde partic est constitué d'une stratification qui alterne minces couches sableuses et niveaux argileux, dont le pendage bascule vers la partic cuvelée en bois, d'ailleurs renforcée sur ce côté. L'hypothèse de l'utilisation de cet aménagement comme bac à décanter/ tremper ou à rincer (lié au travail de la laine ?) a été avancée. Le mobilicr recueilli dans le comblement de cette fosse, dont une applique anthropozoïque en bronze, date l'ensemble du début du III' $s$.

Une portion importante de l'épicarpe d'un fruit de Cucurbilaceae a été trouvée lors du dégagement de cette structure.

Averti rapidement de cette trouvaille tout à fait exceptionnelle, l'un d'entre nous (P. M.) a fait effectuer un prélèvement au sein des sédiments qui subsistaient encore. Un échantillon de sédiment d'un volume de 21 fut alors récupéré par l'équipe de fouilles. Une étude carpologique, plus complète, a donc pu être conduite. Malhcureusement, lors du tamisage un incident technique s'est produit. Un des cribles de la colonne de tamisage s'est renversé et une partie de son contenu a été perdu. Aussi, n'est-il pas envisageable de commenter le nombre de restes attestés. Toutefois, comme l'échantillon s'est révélé assez riche, 28 taxons sont mentionnés, une présentation du cortège des plantes attestées est intéressante, d'autant plus que certaines espèces, à elles scules, justifient un développement, c'est le cas du buis (Buxus sempervirens) et de l'amarante (Amaranthus blitum), sans évoquer le fruit de Cucurbitaceae. 


\section{LES DONNÉES CARPOLOGIQUES}

Vingt-sept taxons sont attestés dans la structure (tabl. I). À part le grain de céréale, tous les carpo-restes sont conservés de manière imbibée.

\section{Les plantes cultivées et/ou exploitées}

Les céréales ne sont représentées que par un seul grain de froment/blé dur (Triticum aestivum/durum), blé très largement exploité à l'époque dans le Bassin parisien. Les cultures jardinées sont marquées par la présence de l'amarante (Amaranthus blitum), du concombre (Cucumis sativus) et de la gourde calebasse (Lagenaria siceraria). Les concombres étaient appréciés dans l'Antiquité. Ils figurent souvent dans les assemblages carpologiques gallo-romains.

Un seul fruitier témoigne de l'arboriculture : la vigne (Vitis vinifera). Une série d'études palynologiques a récemment montré que la viticulture était bien implantée autour de Paris dès le Haut-Empire (Leroyer, 1997).

Même partielles, ces données montrent qu'une agriculture diversifiée (la polyculture) était pratiquée par les habitants de l'établissement romain de Ia Queue de Rivecourt.

La cueillette avait toujours cours comme l'attestent deux végétaux : l'églantier (Rosa sp.) et le sureau noir (Sambucus nigra). Les fruits de ces deux taxons sont du reste couramment collectés et consommés à l'époque romaine.

Le buis (Buxus sempervirens), dont les fruits ne sont pas comestibles, doit certainement correspondre à une espèce ornementale.

\section{Les végétaux spontanés}

Le cortège de plantes spontanées témoigne de plusieurs milieux. Une partie des plantes provient des cultures. Elles ont certainement été apportées en même temps que les végétaux cultivés qu'elles infestaient. Deux plantes sont des mauvaises herbes des champs de céréales automnales: la nielle des blés (Agrostemma githago) et la ravenelle (Raphanus raphanistrum). Cinq espèces correspondent aux cultures sarclées ou de jardin (tabl. I), ce qui montre bien la diversité des productions.

Dans les environs du puits, quelques plantes attestent la présence d'une prairie et de lieux rudéralisés (terrains
Tabl. I - Les végétaux attestés dans la fosse 608 de La Queue de Rivecourt (Oise).

\begin{tabular}{|c|c|c|c|}
\hline \multicolumn{4}{|c|}{ Plantes cultivées ou exploitées } \\
\hline Céréale & Froment & Triticum aestivum s.l. & 1 carbonisé \\
\hline \multirow[t]{3}{*}{ Légumes } & Amarante blette & Amaranthus blitum & 7 \\
\hline & Concombre & Cucumis sativus & 12 \\
\hline & Gourde calebasse & Lagenaria siceraria & 3 fragments \\
\hline Fruitier cultivé & Vigne cultivée & Vitis vinifera & 2 \\
\hline \multirow[t]{3}{*}{ Arbres/arbustes } & Buis & Buxus sempervirens & $1+3$ feuilles \\
\hline & Églantier & Rosa sp. & 1 épine \\
\hline & Sureau noir & Sambucus nigra & 18 \\
\hline \multicolumn{4}{|c|}{ Végétaux spontanés } \\
\hline \multirow[t]{2}{*}{ Cultures d'hiver } & Nielle des blés & Agrostemma githago & 1 \\
\hline & Ravenelle & Raphanus raphanistrum & 2 siliques \\
\hline \multirow{5}{*}{$\begin{array}{l}\text { Cultures sarclées/ } \\
\text { jardins }\end{array}$} & Grande bardane & Arctium lappa & 5 \\
\hline & Chénopode blanc & Chenopodium album & 11 \\
\hline & Chénopode polysperme & Chenopodium polyspernum & 6 \\
\hline & Coqueret & Physalis alkekengi & 2 \\
\hline & Renoncule rampante & Ranunculus repens & 3 \\
\hline \multirow{7}{*}{$\begin{array}{l}\text { Lieux rudéralisés/ } \\
\text { prairies }\end{array}$} & Petite ciguë & Aethusa cynapium & 1 \\
\hline & Petite bardane & Arctium minus & 1 \\
\hline & Arroche astée/étalée & Atriplex prostata/patula & 1 \\
\hline & Lamier blanc & Lamium album & 1 \\
\hline & Grande ortie & Urtica dioica & 1 \\
\hline & Potentille rampante & Potentilla reptans & 4 \\
\hline & Picris fausse-épiaire & Picris hieracioides & 1 \\
\hline \multirow[t]{5}{*}{ Milieux humides } & Plantain d'eau & Alisma plantago-aquatica & 1 \\
\hline & Marisque & Cladium mariscus & 2 \\
\hline & Lycope & Lycopus europaeus & 1 \\
\hline & Grande näade & Najas marina & 1 \\
\hline & Renoncule rampante & Ranunculus repens & 3 \\
\hline
\end{tabular}

vagues, décombres, etc.). Ces milieux sont fréquents autour des habitats humains.

Le caractère humide de l'emplacement est également bien reflété par une série d'autres végétaux : les plantes de milieux humides mentionnées dans le tableau I.

Ces données s'intègrent parfaitement dans le spectre carpologique du gisement, comme l'illustre le tableau II qui présente l'ensemble des plantes cultivées et collectées, reconnues sur le site. Liste qui témoigne de la diversité des espèces cultivées et collectées sur le site. 
Tabl. II - Ensemble des plantes exploitées (végétaux cultivés et cueillis) attestées au Bois Harlé/La Queue de Rivecourt (Oise) : c, carbonisé ; fgt, fragment ; * données quantitatives non fournies (données: A. De Hing, P. Marinval et V. Matterne).

\begin{tabular}{|c|c|c|c|c|c|c|c|c|}
\hline & & & & $\begin{array}{l}\text { La Tène } \\
\text { ancienne }\end{array}$ & Ier / II s. & milieu II $\mathrm{s}$. & début III $^{\text {s. }}$ & $\begin{array}{c}\text { non précisé } \\
\text { De Hingh, } 1993\end{array}$ \\
\hline \multirow{27}{*}{$\begin{array}{l}\text { Plantes } \\
\text { cultivées }\end{array}$} & \multirow[t]{8}{*}{ Céréales } & Froment & Triticum aestivum s.l. & & & $2 c+1$ fgt rachis & $1 \mathrm{c}$ & * \\
\hline & & Amidonnier & Triticum cf. dicoccum & & & & & * \\
\hline & & Blé & Triticum sp. & $2 \mathrm{c}$ & $1 \mathrm{c}$ & $2 \mathrm{C}$ & & \\
\hline & & Orge polystique vêtue & Hordeum vulgare & $3 c$ & & $2 c+4 f g t c$ & & $*$ \\
\hline & & Avoine & Avena sp. & $7 \mathrm{c}$ & & $1 \mathrm{c}$ & & \\
\hline & & céréale & cerealia & & $1 \mathrm{fgt} \mathrm{c}$ & 15 fgt c & & \\
\hline & & Millet commun & Panicum miliaceum & $2 \mathrm{c}$ & & $2 \mathrm{C}$ & & * \\
\hline & & Millet des oiseaux & Setaria italica & $5 c$ & & & & \\
\hline & \multirow[t]{3}{*}{ Légumineuses } & Fèverole & Vicia faba var. minor & $2 c+1 \mathrm{fgt} c$ & & 1 fgt c & & * \\
\hline & & Lentille & Lens culinaris & & & $1 \mathrm{c}$ & & $*$ \\
\hline & & Petit pois & Pisum sativum & & & $2 \mathrm{c}$ & & * \\
\hline & \multirow[t]{6}{*}{ Légumes } & Amaranle blelte & Amaranthus blitum & & & 21 & 7 & \\
\hline & & Bette/Betterave & Beta vulgaris & & & 6 & & $*$ \\
\hline & & Chénopode bon-henri & Chenopodium cf. bonus-henricus & & & 1 & & \\
\hline & & Concombre & Cucumis sativus & & 6 & 2 & 12 & * \\
\hline & & Gourde calebasse & Lagenaria siceraria & & & & & * \\
\hline & & Pourpier & cf. Portulaca oleracea & & & 1 & & \\
\hline & Oléagineux & Pavot & Papaver somniferum & 1 & & 1 & & * \\
\hline & \multirow[t]{4}{*}{ Aromatiques } & Céleri & Apium graveolens & & 12 & $598+2 \mathrm{fgt}$ & & \\
\hline & & Coriandre & Coriandrum sativum & & & & & * \\
\hline & & Fenouil & Feniculum vulgare & & $2+1 \mathrm{fgt}$ & & & \\
\hline & & Moutarde noire & Brassica nigra & & & 9 & & \\
\hline & \multirow[t]{3}{*}{ Fruitiers } & Pin pignon & Pinus pined & & & 1 fruit entier & & \\
\hline & & Prunier crêque & Prunus domestica ssp. insititia & & 3 & & & \\
\hline & & Vigne cultivée & Vitis vinifera & & $15+1 \mathrm{fgt}$ & 4 & 2 & * \\
\hline & \multirow{2}{*}{ Ornementales } & Buis & Buxus sempervirens & & & & $1+1$ feuillle & \\
\hline & & Rosier & Rosa sp. & & & & 1 épine & \\
\hline \multirow{13}{*}{$\begin{array}{l}\text { Plantes } \\
\text { cueillies }\end{array}$} & \multirow[t]{9}{*}{ Arbres/arbustes } & Aubépine à un style & Crataegus monogyna & 1 & 1 & & & \\
\hline & & Cornouiller sanguin & Cornus sanguinea & $3+3 \mathrm{fgt}$ & & 1 fgt c & & \\
\hline & & Églantier & Rosa sp. & & 1 & & & \\
\hline & & Épine-vinette & Berberis vulgaris & & & & & \\
\hline & & Merisier & Prunus avium & & $2+2 \mathrm{fgt}$ & & & \\
\hline & & Noisetier & Corylus avellana & $2+23 \mathrm{fgt}$ & & $8 \mathrm{fgt}$ & & \\
\hline & & Prunellier & Prunus spinosa & $6 \mathrm{fgt}$ & $11+4 \mathrm{fgt}$ & 4 fgt & & \\
\hline & & Sureau hyèble & Sambucus ebulus & 2 & 6 & 1 & & \\
\hline & & Sureau noir & Sambucus nigra & & 14 & $47+5 \mathrm{fgt}$ & 18 & \\
\hline & \multirow[t]{4}{*}{ Herbacées } & Fraisier des bois & Fragaria vesa & & 2 & $3+1 \mathrm{fgt}$ & & \\
\hline & & Framboisier & Rubus idaeus & & 14 & & & \\
\hline & & Mûrier roncier & Rubus fruticosus agg. & 13 & $79+5 \mathrm{fgt}$ & $2+2 \mathrm{fgt}$ & & \\
\hline & & Ronce bleuâtre & Rubus caesius & & 6 & & & \\
\hline
\end{tabular}




\section{Commentaires}

\section{L'amarante blette (Amaranthus blitum)}

L'amarante blette ou blite est une plante annuelle, spontanée en Europe méridionale. Aujourd'hui tombée dans l'oubli, bien que le poète François de Malherbe l'ait chantée comme la rose, elle fut jadis cultivée comme légume vert. À partir de ses feuilles, des tiges tendres et des inflorescences, on préparait des plats d'épinard. On consommait aussi ses graines farincuses, bien qu'elles soient toutes petites (de $1 \mathrm{~mm}$ à $1,5 \mathrm{~mm}$ de longueur). Leur abondance par pied permet de palier le handicap de leur taille et il est très facile d'en récolter rapidement de grandes quantités.

Les Grecs la consommaient, Théophraste l'évoque et des graines figurent dans le sanctuaire d'Héra à Samos (Grèce), daté du VII' s. avant J.-C. (Kučan, 1995). Les textes romains mentionnent également ses divers emplois (Plaute, Pline et Palladius, in André, 1981), mais la plante est jugée insipide. L'amarante est assez. fréquemment retrouvéc dans les niveaux romains et médiévaux d'Allemagne (Körber-Grohne, 1987). En France, pour l'instant, elle n'est signalée qu'à Marseille, dans le niveau hellénistique (III' s. avant J.-C.) des fouilles de la place de La Bourse (Marinval, inédit) et dans un site médiéval (VII'-IX's. après J-C.), Les Ruelles à Serris, Seine-et-Marne (De Hingh, Bakels, 1996).

\section{Le buis (Buxus sempervirens)}

Le buis est un petit arbre très ramifié qui ne dépasse guère 6-8 m de haut. Sa croissance est extrêmement lente mais l'espèce jouit d'une très grande longévité (jusqu'à 600 ans). Son feuillage n'est pas vraiment persistant, mais comme il ne cesse de se renouveler, l'arbre reste toujours vert.

C'est une espèce de demi-ombre qui se rencontre sur les collines sèches et pousse sur sols calcaires. Elle est plutôt thermophile et habite naturellement le sud de l'Europe (espèce supra méditerranéenne). Elle se hasarde peu dans le nord de la France. Dans cette partie septentrionale du pays, son indigénat est d'ailleurs incertain car l'arbre est rarement subspontané, même dans le Bassin parisien (Lambinon et al., 1992).

Le buis ne constitue pas une espèce comestible. Ses usages sont purement décoratifs ou techniques. Son bois est très dur, particulièrement lisse et à grains fins, d'un beau jaune citron. Il se travaille bien. Aussi fut-il souvent utilisé, dès l'Antiquité, en ébénisterie. On confectionnait avec ce bois dense des manches d'outils, des boîtes (les pyxides où l'on enfermait les objets précieux, certains remèdes ou des bijoux) ainsi que des tablettes à écrire. Du reste, des fragments de tablettes à écrire ont été mis au jour sur le site de Longueil-Sainte-Marie. Toutefois, l'un des fragments a pu être déterminé comme étant du pin sylvestre (Pinus sylvestris) (Vangele, Dujardin, 1989).

Cet arbre supporte bien la taille. Dans l'Antiquité romaine, le buis entrait déjà fréquemment dans la composition des jardins d'agrément. Il était taillé et on lui conférait parfois des formes animales allant jusqu'à créer de véritables sculptures végétales (Grimal, 1984).

L'arbre était consacré à Hadès (le dieu des Enfers) et à Cybèle. Il constituait aussi un symbole funćraire en même temps qu'une preuve d'immortalité parce qu'il reste toujours vert (Brosse, 1989, 1990). Les Gaulois auraient, aussi, divinisé le buis qui symboliserait l'éternité (Chevalier, Gheerbrant, 1982). Sur le site de La Queue de Rivecourt, cet arbre est attesté par la présence d'une graine ainsi que par la découverte de trois feuilles. Cellesci sont caractéristiques, de forme ovale à presque ronde. La graine a conservé sa brillance ainsi que l'intensité de sa couleur (noire) et demeure assez coriace.

Le buis devait donc croître à proximité du puits de Longueil-Sainte-Marie, peut-être décorait-il un jardin? En tout cas, il semble que des graines et des feuilles soient tombées naturellement dans la cavité.

En dehors des mentions palynologiques, les découvertes de buis sont rares en archéologie. Des graines sont signalées dans plusieurs sites romains de GrandeBretagne, par exemple à York (Hall, Kenward, 1990) et Stonea Grange (Van der Veen, 1996), ainsi qu'en Allemagne à Cologne (Knörzer, Neu, 1998). Pour la France, il ne s'agit que de la seconde mention de l'espèce. Une feuille a précédemment été retrouvée dans un puits gallo-romain de la région parisienne.

De par son écologie, son aire de répartition et en fonction des croyances et usages antiques, il y a de fortes probabilités pour que cet arbre ait été introduit par les Romains et qu'il se soit acclimaté dans le Bassin parisien. Opinion que partage C. Leroyer (1997) et J.-M. Pernaud (anthracologue, communication orale) qui estiment que le buis n'est pas spontané dans cette région mais d'intro- 
duction récente, sans doute d'époque romaine. En Normandie on a constaté que le buis poussait, en assez. grande concentration, sur et à proximité de sites galloromains, dans des stations où, normalement, l'arbre ne devrait pas être présent puisque le sol est acide (Gidon, 1936). Il pourrait s'agir de reliques d'antiques plantations de l'arbre.

\section{Le rosier (Rosa sp.)}

Outre le buis, une seconde plante ornementale est attestée sur le site : le rosier (Rosa sp.). Il est, en effet, possible d'envisager qu'un rosier agrémentait et ornait cet éventuel jardin, comme le laisse supposer la découverte dans le puits d'une épine de rosier.

\section{La bette/betterave (Beta vulgaris)}

Dans la parcelle du Bois Harlé, A. De Hingh a identifié des graines de bette/betterave (De Hingh, 1993).

La plante cultivée provient de la bette maritime (Beta vulgaris subsp. maritima), végétal vivace qui pousse sur les falaises, les prairies et les dunes sableuses du littoral atlantique et méditerranéen. Apparemment, sa domestication aurait eu lieu au Proche-Orient (Zohary, Hopf, 2000).

Le monde gréco-romain qui cultivait et consommait couramment la bette/betterave distinguait deux types : la bette blanche et la noire. Toutes deux sont des plantes de jardins.

\section{La bette blanche (la Beta candida ou alba de l'Antiquité) ou poirée à cardes}

Les sources littéraires fournissent des informations précises sur sa culture et ses modes de consommation (André, 1981). Les jardiniers romains espaçaient les semis et plantaient les graines lors de trois périodes : au début du printemps, en juin et à l'automne. Procédé astucieux qui assurait une production régulière presque toute l'année (Pline, XIX, 132).

La bette était peu appréciée des Romains nantis qui la considéraient comme un met vulgaire. De ce fait, c'était surtout les classes laborieuses qui en mangeaient (Perse, III, 114 ; Martial, XIII, 13, 1). Pourtant, différents modes de préparation de la bette sont connus. Les bettes pouvaient être accommodées au vin et relevées de poivre comme le propose Martial (XIII, 133, p. 1-21). Apicius les assaisonne aussi fortement (Apicius, III, 11, p. 1-21). Elles étaient rarement consommées seules. Les Anciens les préparaient agrémentées de lentilles ou de fèves et l'on n'oubliait pas de les accommoder de moutarde pour en relever le goût (Pline, XIX, 123; Athénée, 372, a ; Dioscoride, II, 1491).

\section{La bette commune ou ordinaire, la noire (Beta nigra)}

La bette noire possède une racine un peu plus charnue que la forme blanche (Pline, XIX, 132). À partir d'elle, les jardiniers italiens du Moyen Âge sont parvenus à obtenir la betterave. Elle était aussi semée et repiquée à différents moments de l'année (André, 1964).

Pour l'instant, les plus anciennes traces de domestication de l'espèce proviennent de Babylonie. Des bettes étaient ainsi cultivées dans les jardins du roi Merodachbaiadan (722-711 avant notre ère) (KörberGrohne, 1987). La plante est mentionnée dès la transition âge du Bronze/âge du Fer à Troie, Turquie (Riehl, 1999). Puis, elle figure dans différents sites archaïques grecs (datant des $\mathrm{VII}^{\mathrm{e}}-\mathrm{VI}^{\mathrm{e}}$ s. avant J.-C.) : en Grèce, à Samos (Kučan, 1995), ou en Turquie à Aydin (Nesbitt, Samuel, 1996), à Milet, Kalabaktepe et Zeytintepe (Sticka, 1997). En Europe occidentale, la plante ne fait son apparition que dans les niveaux romains. Du fait des orientations de la recherche archéologique, elle est surtout attestée dans les pays nordiques: Allemagne, Grande-Bretagne et Pays-Bas. Par exemple, dix sites romains du Bade-Württemberg en ont livré (Sticka, 1996). En France, elle est signalée dans des horizons du Haut-Empire romain à Auch (Gers) (Ruas, inédit), à Paris (Seine), à Rouen (théâtre des Arts et place Foch) (Seine-Maritime), à Jouars-Pontchartrain (Yvelines) (l'ensemble datant du I ${ }^{\text {er }} s$. après J.-C..) et à Marolles-sur-Seine (Seine-et-Marne) (l'ensemble Matterne, 2001).

\section{La gourde calebasse (Lagenaria siceraria)}

Il ne subsiste environ qu'un tiers de l'extrémité distale d'un fruit de Cucurbitaceae. Les restes du calice de la fleur sont visibles. La partie conservée mesure $136 \mathrm{~mm}$ de long pour une largeur préservée de $50 \mathrm{~mm}$ environ. La forme est subovale. L'épicarpe (la peau du fruit) est relati- 
vement coriace, il présente une épaisseur de 3,5 à $4 \mathrm{~mm}$. Sa surface est lisse ct ne possède pas de côtes apparentes (fig. 9).

La détermination, ne serait-ce qu'au genre de macroreste de Cucurbitaceae, n'est pas aisée à partir de restes de fruits. Les fructifications de cette famille sont très polymorphes et les critères de déterminations spécifiques (fleurs, vrilles, pédoncules des fruits et graines) ne sont pas préservés sur cet exemplaire. Aussi, sommes-nous réduits à n'émettre qu'une proposition déductive d'identification.

Compte tenu des origines biogéographiques (Mathon, 1981), des mentions textuelles (André, 1981) et des découvertes archéologiques (Costantini, 1977, 1979 ; Willcox, 1977 ; Van der Veen, 1980-1981; Kroll, 1982 ; Ruas, 1990 ; Knörzer, 1991), seules quatre espèces de Cucurbitaceae peuvent être présentes en contexte galloromain: le melon (Cucumis melo I..), le concombre (Cucumis sativus L.), la pastèque ou melon d'eau (Citrullus vulgaries Schrad.) et la gourde calebasse (Lagenaria siceraria Standl.). Du fait de l'épaisseur de l'épicarpe, de son aspect coriace et lisse, de l'absence de côtes ou de stries visibles, il est plus vraisemblable qu'il s'agisse d'un fragment de gourde calebasse. La partie préservée n'a, toutefois, pas la forme en poire ou de bouteille caractéristique des calebasses ; mais cette particularité est plus ou moins développée selon les variétés. La gourde des pèlerins a une forme de bouteille; la cougourde possède un goulot allongé tandis que la calebasse est peu étranglée (Candolle, 1883).

L'étude cellulaire, en microscopie photonique, plus précise, confirme cette proposition '. Une coupe pratiquée dans l'épicarpe de l'échantillon montre qu'il est formé d'un épiderme constitué de plusieurs couches de cellules (fig. 10a, b, c). Elles sont de forme irrégulière et de taille réduite, aux parois épaisses (ligneuses) et ponctuées. Il s'agit de cellules sclérifiées de type pierreux. Elles sont similaires à celles observées sur une gourde calebasse actuelle (fig. 10d).

J. André (1981), à la lecture des textes antiques, nous apprend que cette plante était cultivée dans les potagers romains. Séché, son épicarpe servait de récipient. Les données ethnographiques précisent que, dans des temps plus récents, il était utilisé pour contenir des liquides,

1. L'étude cỵtologique a été effectuée par J. Rech du laboratoire d'Alimentation, École nationale vétérinaire de Toulouse.
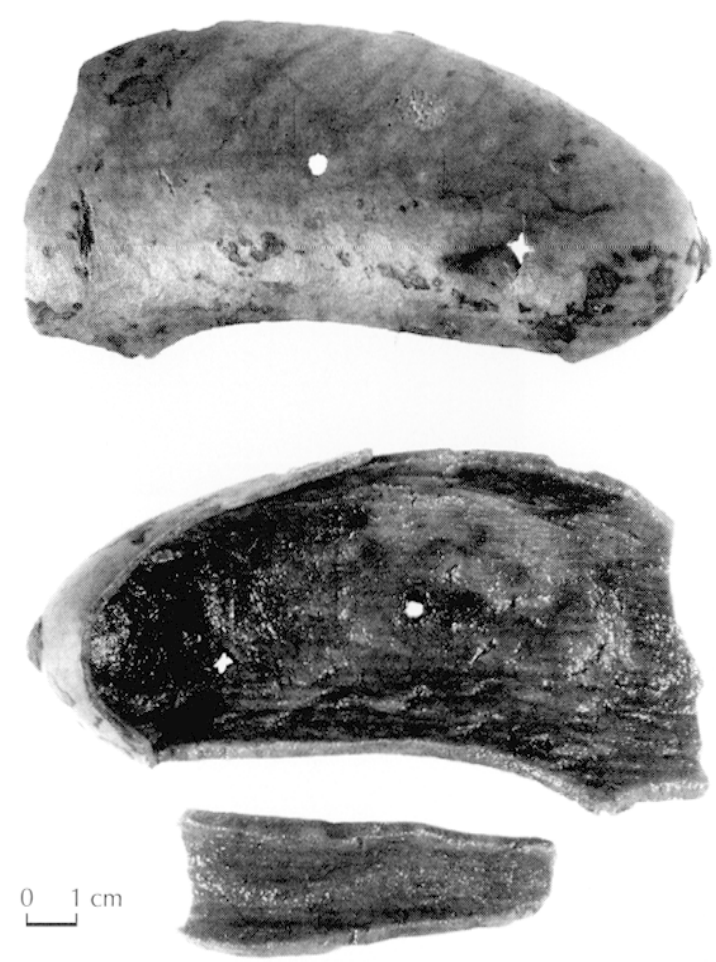

Fig. 9 - Vestiges imbibés de la gourde calebasse (Lagenaria siceraria) de la fosse 608 de La Queue de Rivecourt : vues des deux faces (photo A. Guey, (CNRS).

comme récipient de stockage mais aussi comme ustensile culinaire et comme instrument de musique. Le fruit est considéré par les Romains comme peu nourrissant et figure dans les repas médiocres ou ridicules car sa chair est souvent amère. Toutefois, les fruits verts, non mûrs, sont appréciés. Divers modes de consommation de la gourde sont d'ailleurs indiqués dans les Géoponiques (XII, XIX, XV et XVII) et Apicius fournit neuf recettes dans lesquelles entrent de jeunes fruits immatures (in André, 1981). Ses vertus médicinales étaient reconnues et appréciées dans l'Antiquité, Celse, par exemple, les souligne (livres II et III).

Les Agronomes latins se sont préoccupés des conditions de croissance de la gourde. Pline, Columelle et Palladius fournissent des indications sur la manière de la cultiver et le moment de la récolte (André, 1981). Pline va plus loin en fournissant des précisions sur les modes de conservation du fruit. La gourde était déposée dans une fosse à l'abri du soleil sur une couche de sable et recouverte de foin puis de terre, procédé qui améliore son goût. On la conservait aussi dans la saumure (Pline, H. N., XIX, 74). 

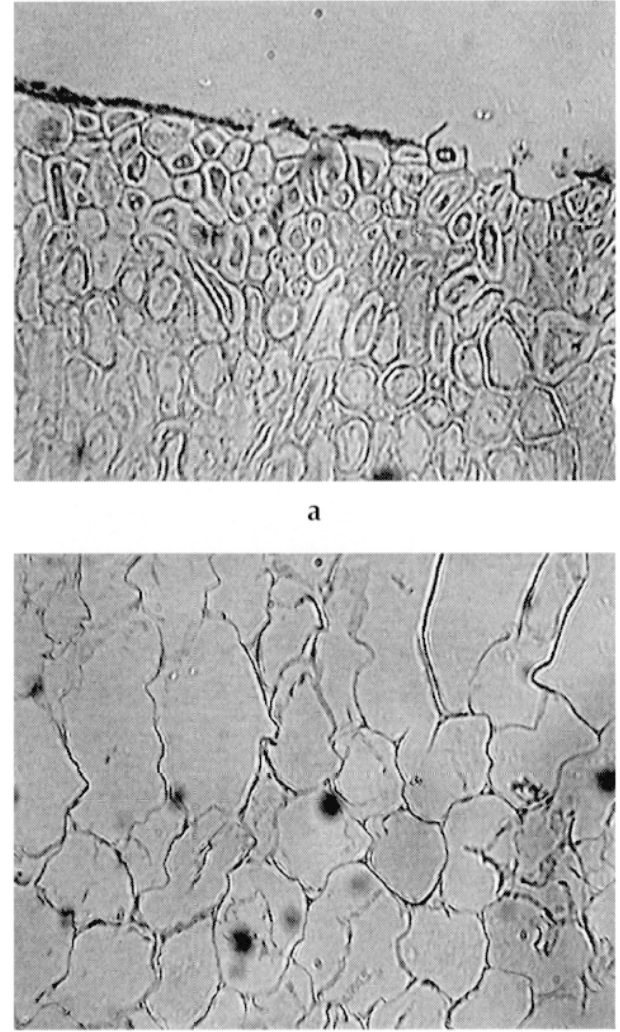



b

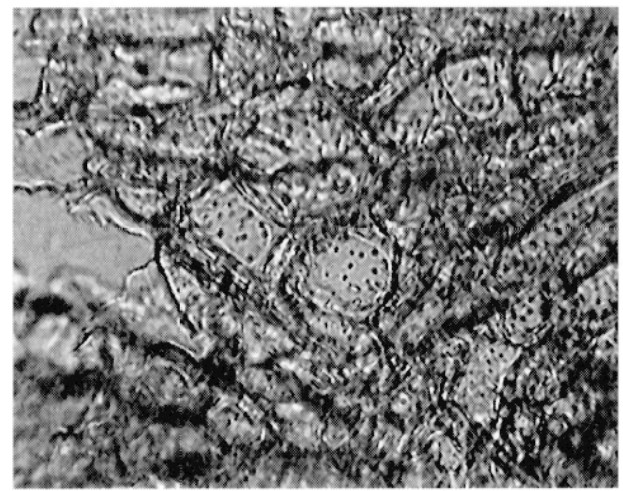

d

Fig. 10 - Coupe microscopique de l'échantillon archéologique de la gourle calebasse : a, b, coupes de l'épiderme ; c, coupe au sein de la paroi ; d, coupe microscopique d'une gourde calebasse actuelle (photo J. Rech, ENV).

La gourde calebasse a surtout été cultivée pour la particularité des formes que revêt le fruit et sa capacité à durcir à maturité, donc à se conserver, ainsi qu'à devenir parfaitement étanche aux liquides. En suspendant les pousses on peut même modeler à loisir la forme des fruits, notamment en les introduisant dans des étuis d'osier après la floraison. Dans l'Antiquité, les gourdes calebasses avaient de multiples emplois : récipients (pour contenir de l'eau, du vin et même stocker du miel ou de la poix...), bols pour manger ou boire, flotteurs pour les filets de pêche, boućes pour les enfants qui apprennent à nager, instruments de musique... (André, 1981).

La partie conservée de l'épicarpe de la gourde calebasse de La Queue de Rivecourt possède deux perforations qui sont distantes de $39 \mathrm{~mm}$ (fig. 11). La première est subcirculaire et présente un diamètre maximal de $3 \mathrm{~mm}$ et la seconde possède une section plus ou moins carrée à cruciforme de $1,5 \mathrm{~mm}$ à $2 \mathrm{~mm}$ (les mesures sont prises sur le côté interne du fruit). Elles furent réalisées de l'extérieur vers l'intérieur comme en témoigne l'observation des parois des perforations (celles-ci présentent une inclinaison de l'extérieur vers l'intérieur) et le cône d'impact observable sur la paroi interne du fruit. Les surfaces de fractures sont altérćes. Aussi, ces perforations apparaissent-elles comme anciennes, mais nous ignorons leur origine et leur fonction. Tout au plus est-il possible de supposer que si la calebasse était perforée de la sorte lors de son utilisation, elle ne devait donc pas assurer une fonction de stockage d'un liquide.

L'espèce est originaire d'Afrique tropicale ou d'Afrique du Sud. À part l'Afrique du Sud où elle est signalée vers 2000 avant notre ère (Davies, 1975), c'est en Égypte que l'on rencontre les plus anciennes mentions. La gourde calebasse est attestée dans les tombes égyptiennes au cours du $\mathrm{I}^{\text {er }}$ millénaire avant J.-C. (Germer, 1985) . Par la suite, c'est essentiellement en Gaule romaine qu'elle est mentionnée. Des graines provien-

2. Bien que des fruits soient mentionnés très tôt vers 3500-3000 avant J.-C. (Schweinfurth, 1912), il conviendrait de réexaminer le contexte de ces découvertes. 




a

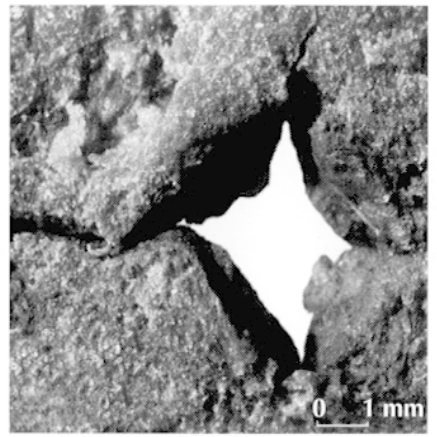

b



C

Fig. 11 - Perforations sur la gourde calebasse de la fosse 608 de La Queue de Rivecourt. Une perforation (a et b) est cruciforme, l'autre (c) est ronde (photo J. Rech, ENV).

nent de plusieurs sites romains d'Allemagne: HanauSalisburg et Kesselstadt, Lahr, Hesse et Dieburg (voir par exemple: Küster, 1995; Göldner, Kreuz., 1999). Un exemplaire douteux (Lagenaria cf. siceraria) figure dans le comblement d'un puits daté de la seconde moitié du II's. après J.-C. à Mazières-en-Mauges, Maine-et-Loire (Ruas, 1990) ; sans oublier les deux exemplaires qui ont été retrouvés dans un puits de la fin du $\mathrm{II}^{\circ} \mathrm{s}$. ou du début du III" s. après J.-C. dans la parcelle du Bois Harlé à Longueil-Sainte-Marie (De Hingh, 1993). Il est d'ailleurs curieux de constater que ce ne sont, pour l'instant, que des sites localisés dans la moitié nord du pays qui ont livré des vestiges de gourde calebasse.

Comme on peut le constater, les mentions archéologiques de graines de gourde sont rares, celles de fruits entiers ou de fragments d'épicarpes de Cucurbitaceae le sont plus encore. À notre connaissance, il s'agit de la troisième attestation de restes de fruits. Deux courges entières (dont l'identification serait à préciser) ont été signalées au XIX"s. dans une tombe du cimetière alémanique du mont Lupfen à Oberflacht (BadeWürttemberg, Allemagne) où les conditions de conservation sont exceptionnelles (Durrich, 1849, in Salin, 1959 ; Paulsen, 1992 ; Schiek, 1992). À Amsterdam (PaysBas), des fragments de péricarpe ont été récupérés dans un puits daté également des débuts du haut Moyen Âge (vers 650-740 après J.-C.) (Kooistra, Hessing, 1989). De menus morceaux figurent aussi dans les sédiments du village lacustre du XI" s. du lac de Paladru à Colletières (Charavines, Isère) (Lundström-Baudais et al., 1993).

\section{Une espèce exotique importée, peutêtre pas?}

La gourde calebasse est une espèce tropicale. Du fait de ses origines, elle réclame des climats chauds et ne supporte absolument pas les gelées nocturnes. Aussi, ne croît-elle, en général, qu'en région méditerranéenne. Toutefois, sa culture dans le Bassin parisien ne peut être exclue, bien que pour certains auteurs la plante ne puisse fructifier sous des climats frais (Brouwer, Stählin, 1953). Vilmorin-Andrieux précise pourtant que les gourdes parviennent à mûrir sous le ciel de Paris à l'aube du $\mathrm{XX}^{\mathrm{c}}$ s. Il convient de les semer en un lieu bien exposé à la chaleur du soleil et préservé des courants d'air précise-t-il (Vilmorin-Andrieux et al., 1904). De surcroît, les descriptions précises que fournit Walafridus ainsi que les conseils qu'il donne dans le Carlulaire de Villis (daté de 800 après J.-C.) suggèrent même, très fortement, que l'espèce est cultivée jusque sur les rives du lac de Constance (Bade-Württemberg, Allemagne) (Kooistra, Hessing, 1989). Ces auteurs ont d'ailleurs tenté l'expérience de semer des graines de gourde dans un jardin urbain d'Allemagne du Nord. Ils ont montré à cette occasion que, lors d'un été chaud, la plante peut non seulement pousser mais fructifier (Kooistra, Hessing, 1989). Dans ces conditions, pourquoi ne pas envisager qu'à l'époque romaine la gourde calebasse croissait sur les bords de l'Oise? On a pu la cultiver dans un endroit ensoleillé, bien protégé, à l'abri du vent et du froid. Les découvertes en deux emplacements du même site et la présence concomitante de graines et de restes de fruits vont, d'ailleurs, plutôt dans 
le sens d'une culture locale. Dans cette optique, on peut signaler que plusieurs fosses à fond plat et parois abruptes, pauvres en vestiges, pourraient s'apparenter à des châssis. Le sable nécessaire se trouve juste dans le sol sous-jacent (Maréchal, inédit). Renforçant l'idée de cultures jardinées, il faut souligner que, parmi le peu de mobilier métallique retrouvé, trois fers de houes et un de bêche ont été mis au jour sur le site (Vangele, Dujardin, 1989).

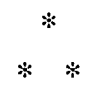

Malgré le caractère, que certains pourront juger anecdotique, de ces découvertes nous avons choisi de les présenter car elles sont suffisamment exceptionnelles pour mériter une large diffusion. Les attestations de cônes complets (ou presque) de pin pignon et de fruits de Cucurbitaceae ne sont pas fréquentes en Europe occidentale. Les mentions de gourde calebasse, d'amarante et de buis sont encore plus rares.

Outre leur aspect spectaculaire, ces découvertes possèdent une valeur historique indéniable. Les dates d'apparition de certains taxons sont ainsi mieux cernées ainsi que les courants d'échange. Les dynamismes de l'horticulture et du commerce romains sont mis en relief. Des denrées méditerranéennes "fraîches " peuvent maintenant être utilisées, consommées en Gaule du Nord. Des plantes méditerranéennes peuvent même être mises en terre. Lors des périodes antérieures, il semble que seuls des produits transformés, comme le vin, étaient importés et qu'aucune plante exotique, de climats chauds, n'était cultivée en Gaule chevelue. Une précision est aussi apportée sur le commerce des productions de pin pignon. Les découvertes de cônes complets ou de fragments aussi bien en Grande-Bretagne qu'en Armorique et dans la vallée de l'Oise, comme leur présence à bord de navires romains attestent la commercialisation d'un produit relativement brut sur de longues distances. Ce sont donc les cônes qui voyageaient et étaient vendus. Les pignons n'étaient apparemment pas extraits sur les lieux de production, afin de réduire le volume à transporter, mais au gré des besoins lors de la consommation.

Ces précisions ainsi que les contextes dans lesquels certaines découvertes eurent lieu (monde religieux) renforcent la valeur intrinsèque (cultuelle, culturelle...) des cônes de pin pignon.

Ces attestations témoignent aussi de l'intérêt et de la richesse de structures en milieu humide. Il est impératif qu'un programme d'analyse des vestiges périssables soit élaboré au plus vite, notamment dans les régions ou pour des périodes pour lesquelles ce genre d'analyse n'est pas pratiqué, afin que ce patrimoine et les informations scientifiques qu'il recèle ne soient pas complètement détruits et perdus. 


\section{BIBLIOGRAPHIE}

A.NDRÉ.J.

1964 : Pline l'Ancien, Histoire naturelle, liwe $X I X$, Paris, Les Belles Lettres.

1981 (1'éd. 1961) : L'alimentation et la cuisine à Rome, Paris, Les Belles Lettres.

BAKFLS C. C.

1991 : "Western Continental Europe", in VAN ZFISI W., Wastiliona K., Bentre. K.-E. (eds), Progress in Old World Palaeoelhnobolany, Rotterdam, Balkema, p. 279-298.

BHRNARI V., DiFTRICII A.

1990 : "I.es objets en matières orgatniques découverts sur le site du "Bois Harlé" à Longueil-Sainte-Marie (Oise) ", in Valtarin B., PRodr:o) F. (dir.), Fouille dans les sablierres de la moyenne vallée de l'oise en 1989, $\mathrm{n}^{\circ}$ spécial 8 à la Rryue arhéologique de Picardie, p. 147-158.

Bouby L., Marinial. P.

2000: "Ressources végétales à Marseille et dans les sociétés indigènes au Bronze final et au Premier Âge du Fer ", in Janin T. (éd.), Mailhac et le Premier $\hat{A} g e$ du Fer en kurope occidentale. Hommages à (). et J. Taffanel, Actes du colloque international de Carcassonne, 17-20 sept. 1997, Monographies d'archéologie méditerranéenne, 7, p. 205-214.

Brosste J.

1989 : Mythologie des arbres, Paris, Plon. 1990 : Les arbres de France: histoire et légendes, Paris, C. de Bartillat.

Brol Wer W., Stählis A.

1953 : Handbuch des Samenkunde für landwirlschaft, Gartenbau und Forstuirtschaft, Frankfurt/Main, DLG; Verlag.

(ANI)(II. A. DF.

1883: Origine des plantes cultivéps, Fac similé 1984, Marseille, Jeanne Lafitte.
Cimatimer J., Ginerkbrant A.

1982 : Dictionnaire des symboles. Mythes, rêes, coutumes, gestes, formes, figures, couleurs, nombres, Paris, Robert Laffont, coll. Bouquins.

\section{Collectif}

1980 : Saintes, cité bimillénaire. Dix ans de recherches archéologiques, Catalogue de l'exposition, Saintes, $1^{\mathrm{cr}}$ juin-l $\mathrm{l}^{\mathrm{cr}}$ oct. 1980, Saintes, Ville de Saintes.

\section{Costantini L.}

1977 : "Le piante ", in Tucial G. l:T Al. (eds), La città bruciata del deserto salato, Mestr (Venezai), Erizzo, p. 159-171.

1979 : " Plants remains at Pirak, Pakistan ", in JARrice J.-F., Sintoni M. (éds), Fouilles de Pirak, 1, Paris, Publications de la commission des fouilles archéologiques, p. 326-333.

Colion G., Giralite J.-L., Bourin J.-Y., Marinval. P.

1985 : «Les bains et l'environnement de la villa gallo-romaine de la Pétonnière à Paulnay (Indre) ", Rerue archéologique du Centre de la France, 24, 2, p. 191-214.

DAIIES F.

1975: "Excavations at Shongwei South Cave : the oldest evidence to date for cultigens in Southern Africa ", Annals of Natural Museum, 22, p. 627-662.

DE HINciH A.

1993 : «Bottle gourd seeds at GalloRoman "Le Bois Harlé" (Oise, France) ", Analecta Praehistorica Leidensia, 26, p. 93-97.

Di: Hiscil A., Bakils C.

1996 : «Palaeobotanical evidence for social difference?, the example of the Early Medieval domain of SerrisL.es Ruelles, France ", Vegetation History and Archaeobolany, 5, 1-2, p. $117-120$.
DF: Hixgih A., KoOISTRA L.

1995 : "Reste von getreide und anderen Pflanzen ", in Hadi.bos J. K. (ed.), Castra und Canabae, Ausgrabungen auf dem Huneberg in Nijmegen, 1987-1994, p. 103-109.

Ghenter R.

1985 : Flora des pharaonischen Ägypten, Mainz/Rhein, P. von Zabern.

(GIION F.

1936 : "Résidus de la flore alimentaire médiévale au Mont-Saint-.Michel et sur le littoral de la campagne de Caen : anciennes dunes et anciens vignobles de dunes ", Bulletin de la Sociélé des antiquaires de Normandie, 1936, p. 290-309.

Girird M., T(hernia A.

1978: "Remarques à propos des cônes de pin pignon (Pinus pinea) découverts sur l'épave de la Madrague de Giens ", in T(hlekna A., Pomfy P., HesNARI) A. (dir.), L'épaze romaine de la Madrague de (iiens (Var), 34 Suppl. à Gallia, Paris, éd. du CNRS.

GÖIIDNTR H., KREL\% A.

1999 : "Begraben an cinem "stillen Ort" : botanische und archäologische Funde als neue Hinweiser zum I.eben im römischen Dieburg ", Denkmalpflege und Kulturgeschichte, 2, p. 10-17.

GREEN F. J

1981 : "Iron Age, Roman and Saxon Crop: the archaeological evidence from Wessex ", in Jones M., Diмbi.fiy G. (eds), The environment of man: the Iron Age to the Anglo-Saxon Period, British Archaeological Report, international series, 87 , p. $129-153$.

Greic; J. R. A.

1991 : "The Britisch Isles ", in Vax ZFIST W., Wastr.IKOW K., Behrl: K.-E. (eds), Progress in Old World 
Palapoethnobotany, Rotterdam, Balkema, p. 299-334.

(iRIMAI. P.

1984 : Les jardins romains, Paris, Fayard.

HAI.I. A. R., KeNWARD H. K.

1990 : “Environmental evidence from

the Colonia : general accident and Rougier street ", Archaeological York, 14, 6, p. 289-434.

\section{KISI.FY M. E.}

1988 : "Pinus pinea in agriculture, culture and cult ", in Küsrikr H. (ed.), Der prähistorische Mensch und seine Umwell, Stuttgart, Konard.

\section{KNÖRZER K. H.}

1991 : « Deutschland nördlich der Donau ", in VAN ZEIST W., WASYlikoWA K., BFHRF. K.-E. (eds), Progress in Old World Palaeoethnobotany; Rotterdam, Balkema, p. 189-206.

KNÖ̈RFR K. H., NFU S.

1998 : "Archäologische Unterschungen an der Pectrudengasse in Köln ", Köln Jahrlnïcher, 31, p. 445-480).

KoOIstra L. I., Hzssinc; W. A. M. 1989 : " A tropical surprise in a Dutch Early Medieval well ", Archüobotanik. Dissertationes Bolanicae, 133, p. 167-174.

KÖRBRR-GROHNE: U.

1987 : Nulzpflanzen in Deutschland, Kullurgeschichte und Biologie, Stuttgart, K. Theiss.

\section{Krol.I. H.}

1982 : “ Kulturpflanzen von Tiryns ", Archäologischer Anzeiger, 1982, p. $467-485$.

KLČAN D.

1995 : "Zur Ernährung und dem Gebrauch von Pflanzen im Heraion von Samos im 7. Jahrhundert v. Chr. ", Jahrbuch des Deutschen Archäologischen Instituts, 10, p. 1-64.

Kïstr:R $\mathrm{H}$.

1995 : «Weizen, Pfeffer, Tamnenholz.
Botanische Untersuchungen zur Verbreitung von Handelsgütern in römischer Zeit ", Münster Breit. Ant. Handelsgütern, 14, 2, p. 1-26.

LambiNon J., LaNGiHE J.-E. DF, Dlilvosali.e L., DLvigneaud J. kT al. 1992 (4`éd.) : Nouvelle flore de la Belgique, du Grand-Duché du Luxembourg, du nord de la France et des régions voisines, Meise, éd. du Patrimoine du Jardin botanique national de Belgique.

LaMBOGIIA N.

1952 : "La nave romana di Albenga ", Rivista di Studi Liguri, 18, 3/4, p. 131-236.

\section{Lepetz S., Maréchal. D.}

1996 : “Organisation et fonction du village du Haut-Empire de LongueilSainte-Marie (Oise) et de son parcellaire ", in Cholquik G. (dir.), Les formes du paysage -1- Eitudes sur les parcellaires, Paris, éd. Errance, p. 57-82.

\section{LEROYER C.}

1997 : Homme, climat, végétation au Tardiet Postglaciaire dans le Bassin parisien: apports de l'élude palynologique des fonds de vallée, thèse de doctorat, univ. de Paris 1.

Lignerletx Y., Peters J., Ruas M.-P., Sillitikes $P$.

1997 : «Le culte d'Isis en Bétique ", L'archéologue, Archéologie nouvelle, 28, p. $41-45$.

Lunidström-Batdias K., Mignot C. avec la collab. de Brunitr C., Grtider A., Baudats D., Bidiult M.

1993 : « Le milieu végétal au XI" siècle : macro-restes et paléo-semences ", in Colatrdelate M., Verdet. E. (dir.), I,es habilats du lac de Paladru (Isère) dans leur environnement. La formation d'un terroir au XI' siecle, Document d'archéologie française, Paris, éd. de la Maison des sciences de l'homme, 40, p. 77-97.

\section{MARINIAL P. P.}

1992 : “Céréales, fruits et légumes : les données de l'archéobotanique ", in
Blanc: N., Nerchssian A. (dir.), la cuisine romaine antique, Dossiers d'archéologie, hors série, 3, Glénat, Faton, p. 34-40.

1993 : "Études carpologiques d'offrandes alimentaires végétales dans les sépultures gallo-romaines : réflexions préliminaires ", in FFrdiÈr: A. (dir.), Monde des morts, monde des vivants en Gaule rurale, Actes du colloque archea/ager, Orléans, 7-9 fév. 1992, 6r Suppl. à la Rerne archéologique du Centre de la France, p. 45-65.

Mathon C.-C.

1981 : L'origine des plantes cultivées, Phylogéographie appliquée, Paris, Masson.

\section{MATTERnE V.}

2001 : Agriculture et alimentation vieggétale durant l'âge du fer et l'époque galloromaine en France septentrionale, Archéologie des plantes et des animaux, 1, Montagnac, Monique Mergoil.

MEYER F. G.

1980 : "Carbonised food plants of Pompei, Herculanum and the villa at Torre Annunziata ", Economic Bolany, 34 , p. 401-437.

Nisbitt M., SAMLEL D.

1996 : “ Archaeobotany in Turkey: a review of current research ", OrientLxpress, 3, p. 91-96.

PAuLse. P.

1992 : Die Holzfunde aus dem Gräberfold bei Oberflacht und ihre Kullurhistorische Bedeutung, Stuttgart, Theiss.

Pinard) E., Collate J.-L., Maldain F., MARÉ,:HAI, D.

1999 : “De l'architecture à la hiérarchisation sociale du $V^{c}$ s. av. J.-C. au III" s. apr. J.-C. dans la moyenne vallée de l'Oise (Oise) ", in Brazmer F., Ciliziou S., Coldart A. (dir.), Habitat el sociélé, Actes des XIX" Rencontres internationales d'archéologic et d'histoire d'Antibes, 22-24 oct. 1998, Antibes, APDCA, p. 363-382. 
Pissot V., Vacigele F, Marf́chal D. 1994 : " Longueil-Sainte-Maric "La Queuc de Rivecourt"-"Le Bois à Bouleaux" ", Bilan scientifique 1993 Picardie, Direction régionale des Affaircs culturclles, Service régional de l'archéologie, Amiens, p. 93-95.

Pradat B.

1994 : Ies offrandes alimentaires végétales dans les tombes antiques : exemples d'incinérations à Lyon el à Marseille, mém. de DEA, univ. de Paris 1.

RIEHI, S.

1999 : "Archäobotanik in der Troas ", Stud Troica (Mainz), 9, p. 367-409.

Roi. R., Toulgovat P., Jacamon M. 1981 : Flore des arbres et arbrisseaux, Paris, La Maison rustique.

RLAS M.-P.

1990 : Recherches carpologiques dans le Massif armoricain du Mésolithique au bas Moyen Âge, mém. de DEA, EHESS, Paris.

SAIIN E.

1959 : La civilisation mérovingienne -4les croyances, conclusion, Paris, Picard.
ScHilk S.

1992 : Das Gräberfeld der Merowingerzeit bei Oberflacht, Stuttgart, K. Theiss.

SchueINfuRTH G.

1912 : Arabische Pflanzennamen aus Aegypten Algerien und Jemen, Berlin, D. Reimer.

STrCKA H. P.

1996 : « Römerzeitliche Pflanzenreste aus Baden-Württemberg ", Materialhefte zur Archäologie in BadenWürttemberg, 36, Stuttgart, K. Theiss.

1997 : Pflanzenreste aus dem archaischen Milet. Vorbericht zur Kampagne 1992, in GRAEVE. V. VON (ed.), « Milet 1994-1995 », Archäologischer Anzeiger, p. 157-163.

VAN DER VLEN M.

1980-1981 : «The Ghirza plant remains : Romano-Libyan agriculture in the Tripolotanian pre-desert (appendix II) ", in Barker G., Jonf.S G. (eds), The UNESCO Libyan Valleys Survey 1980, Libyan Studies, $12^{\text {th }}$ Annual Report of the Society of Libyan Studies, p. 45-48.

1996 : "Plant remains ", in JACKSON R. P. J., Potter T. W. (eds),
Excavations at Stonea, Cambridgeshire 1980-1985, London, Bristish Muscum, p. 613-639.

Vanglite F., Dejakdin J.-C.

1989 : Longueil-Sainte-Marie " le Bois Harlé ", bourgade agricole gallo-romaine. Archéologie 88, fouilles et sauvelages dans la moyenne vallée de l'Oise, Chevrières, CRAVO, p. 44-56.

VII.MORIN-ANDRIFux H. IT al.

1904 : Les plantes potagères. Descriptions et cultures des principaux légumes des climats tempérés, Paris, Les Éditions 1900.

Willcox G.

1977 : "Exotic Plants from Roman Waterlogged Sites in London ", Journal of Archaeological Science, 4, p. 269-282.

\section{ZOHARY D., HOPF M.}

2000 (1 $1^{\text {re }}$ éd. 1988) : Domestication of Plants in the Old World. The origin and spread of cultivated plants in West Asia, Europe, and the Nile Valley, Oxford, Sciences Publications. 